\title{
P7: Pflegekammer-Diskussion in Deutschland - Eine kritische Literaturanalyse
}

\author{
Kathrin Knuth
}

Online publiziert: 30 . Oktober 2013

(C) Springer-Verlag Wien 2013

Hintergrund: Die Debatte um deutsche Pflegekammern wird seit längerer Zeit geführt. Unsere Projektgruppe ging der Frage nach, welche Argumente dafür und welche dagegen diskutiert werden und wo Deutschland im europäischen Vergleich steht. Ziel war es zu klären, inwieweit Elemente nationaler und internationaler Kammern und kammerähnlicher Institutionen auf eine Pflegekammer übertragbar wären.

Methode: Die Fragestellungen wurden auf der Basis der Auswertung einer umfangreichen Literaturrecherche diskutiert, die von April bis Juni 2013 im deutschen, englischen und polnischen Sprachraum stattfand. Auszüge französischer, spanischer und ungarischer Literatur wurden - gezielt übersetzt - einbezogen. Aufgrund mangelnder Literatur in gedruckter Form stützte sich die Suche hauptsächlich auf Internetportale. Ergänzend wurden Telefonate und persönliche Gespräche geführt.

Ergebnisse: Die Literaturbewertung ergab: Es existieren viele politische Stellungnahmen und wenig zitierfähige Literatur und wissenschaftliche Studien, welche jedoch im Ergebnis pro Pflegekammer ausfielen. Auf der Grundlage der Rechercheergebnisse wurden Kammern des deutschen Kammersystems mit entsprechenden Institutionen der Pflege in Europa verglichen. Der Focus lag auf zentralen Merkmalen, Organisationsstruktur und Aufgaben von Kammern. Im Ergebnis zeigte sich: Es gibt in Europa derzeit keine einzige reine Pflegekammer, sondern ausnahmslos Kammern, die mehrere Heilberufe vereinen oder andere kammerähnliche Strukturen in politischer und/oder fachlicher Abhängigkeit von Parteien, Regierungen oder anderen Berufsgruppen.

Schlussfolgerung: Eine Pflegekammer in Deutschland könnte demnach die europaweit erste Kammer sein, die ausschließlich Pflegeberufe vereint. Weiterführend gilt es für uns herauszufinden, wie eine Pflegekammer gestaltet werden müsste, um auf vielen Ebenen von Nutzen zu sein. Dabei erscheint die Übertragung einzelner Elemente bestehender Kammern auf Pflegekammern als Möglichkeit die Attraktivität des Pflegeberufes und die Qualität der Pflege positiv zu beeinflussen.

\footnotetext{
K. Knuth $(\bowtie)$

Projektgruppe „@projektforscht“, Gesundheits- und Pflegemanangement, Alice-Salomon Hochschule, Berlin, Deutschland

E-Mail: FranziskaMzS@gmx.de
} 